\title{
User Interface to Express Kinetic Typography with Emotion in Mobile Instant Messengers
}

\author{
Jung Seung-Ah ${ }^{1}$, Cho Yoon $\mathrm{A}^{2}$ and Soon-Bum Lim ${ }^{3}$ \\ Dept. Multimedia Science, Sookmyung Women's University, Korea \\ Itmddkjung@naver.com, ${ }^{2}$ fanwith@naver.com, ${ }^{3}$ sblim@sookmyung.ac.kr
}

\begin{abstract}
To overcome the limitation of emotional transmission with traditional text-based instant messaging systems, this paper designs a messenger user interface for transmitting emotion using kinetic typography. This interface provides two input methods to apply motions to the character. One is 'select emotion after selecting motion' method interface, the other is 'select motion after selecting emotion' method interface. Each method provides a basic motion library, nine parts of emotion coordinates, and a library for adding emotion easily. These provide parameter value control function for modifying more appropriate motion with user's feelings. In other words, a user can transmit entered text with appropriate emotion by using basic motion library and emotion library together, and control parameter values. Additionally, researchers have confirmed the need of the proposed user interface's two input methods, 'select emotion after selecting motion' and 'select motion after selecting emotion', through evaluation of serviceability. With the proposed mobile instant messenger, kinetic typography that transmits emotion easily and quickly can be produced.
\end{abstract}

Keywords: kinetic typography; emotional user interface; motion library; emotional matrix

\section{Introduction}

Text has been used as the most important communication method to exchange information in web environments. However, the delivery of static text in a text-based communication has limitations in delivering the emotion compared to direct dialogue. So, emoticons were used to deliver the emotion. Emoticons are used to represent the user's feelings by using a variety of expressions. However, it doesn't reflect the difference between different emotions with different people since the service is pre-manufactured. Kinetic typography, which adds temporal properties and color, size, position into static text, can be a method to convey additional information with text. Furthermore, kinetic typography includes emotion into transitional text, thus makes people transfer the emotions and feelings more accurately rather than traditional text-only communications [1]. In order to produce kinetic typography, we have to use PC-based professional animation such as Adobe After Effects and Premiere, or programming language such as SVG. However, it is not suitable as a method to manufacture kinetic typography in mobile instant messenger application because it takes a long production time as well as it requires experts with specialized skills. In this paper, researchers have designed the emotional user interface for manufacturing emotional kinetic typography as adding the desired emotion into the motion with text. 


\section{Related Research}

\subsection{Messenger Using Kinetic Typography}

Kinetic typography can be utilized as a mean for delivering emotion effectively as applying the emotional kinetic motion [2]. The created kinetic effects can be utilized in real time communications and can make the expression be richer by controlling the properties of the motions. But all the effects could never be included in the library.

Therefore, we analyzed the sample movements contained in the After Effects presets and defined the movement and properties of the underlying units. A combination of unit movements and kinetic properties make the various kinetic typography effects. The researchers propose an emotional user interface based on the kinetic messenger system, which makes users utilize kinetic typography effects in real time mobile instant messenger communications [2].

\subsection{Messenger Using Kinetic Typography}

We propose a kinetic motion API that will enable various movements to utilize kinetic typography in the application. Kinetic motion API is defined according to the fundamental level of controlling the geometric transformation of the text movement [3]. Kinetic motion API is generally classified into primitive motion API and composite motion API, which have subdivided motion and its attribute value. It provides a function of motion that should construct a motion library that includes a font-rendering module to show a vector font applied to the motion effect on the screen.

According to the motion classification of kinetic typography, primitive motion API is classified into three categories that are static, linear, and curve motion. Static motions include static shaking, static rotation, static scaling, and static opacity. Kinetic Motion API allows kinetic typography to be utilized in diverse applications by replacing the existing methods that express kinetic typography. Users can make a motion library using kinetic motion API to utilize in diverse applications by replacing the existing methods.

\section{Design of Emotional User Interface}

In this paper, researchers have designed an emotional user interface that presents a method for production and utilization according to the user's feelings in mobile instant messengers. This user interface provides two input methods: 'Select emotion after selecting motion' method and 'Select motion after selecting emotion' method. 'Select emotion after selecting motion' method is where a kinetic typography effect is applied when the user selects a motion and then selects an emotion. 'Select motion after selecting emotion' method, on the other hand, is where a kinetic typography effect is applied when the user selects an emotion and then selects a motion. 'Select emotion after selecting motion' method provides a basic motion library that includes six motions that can represent the user's feeling. Accordingly, the emotion library provides the selected motion divided by the intensity of the nine sections, which provide service for expressing kinetic typography. 'Select motion after selecting emotion' method provides an emotion library that divides the user's feelings by the intensity of the nine sections and provides a motion library for the feeling selected by user.

\subsection{Messenger Using Kinetic Typography}

According to the study of emotions through kinetic typography [4], emotions can be classified along with mood and energy. The emotion matrix is configured by $x$-axis and $y-$ axis; the $\mathrm{x}$-axis specifies the energy and the $\mathrm{y}$-axis indicates the mood. The quadrants from $\mathrm{A}$ to $\mathrm{P}$ were divided into 16 sections that express the feelings to express emotion representative. 
In this paper, we propose an emotion matrix that also can be classified along with mood and energy. The x-axis is energy and y-axis is mood. The 16 sections with marked feelings of emotion passed through kinetic typography are reduced to 9 sections to take into size of the mobile screen. The emotion matrix is configured by $\mathrm{x}$-axis and $\mathrm{y}$-axis; the $\mathrm{x}$-axis indicates the mood and the $\mathrm{y}$-axis specifies the energy as shown in Figure 1.

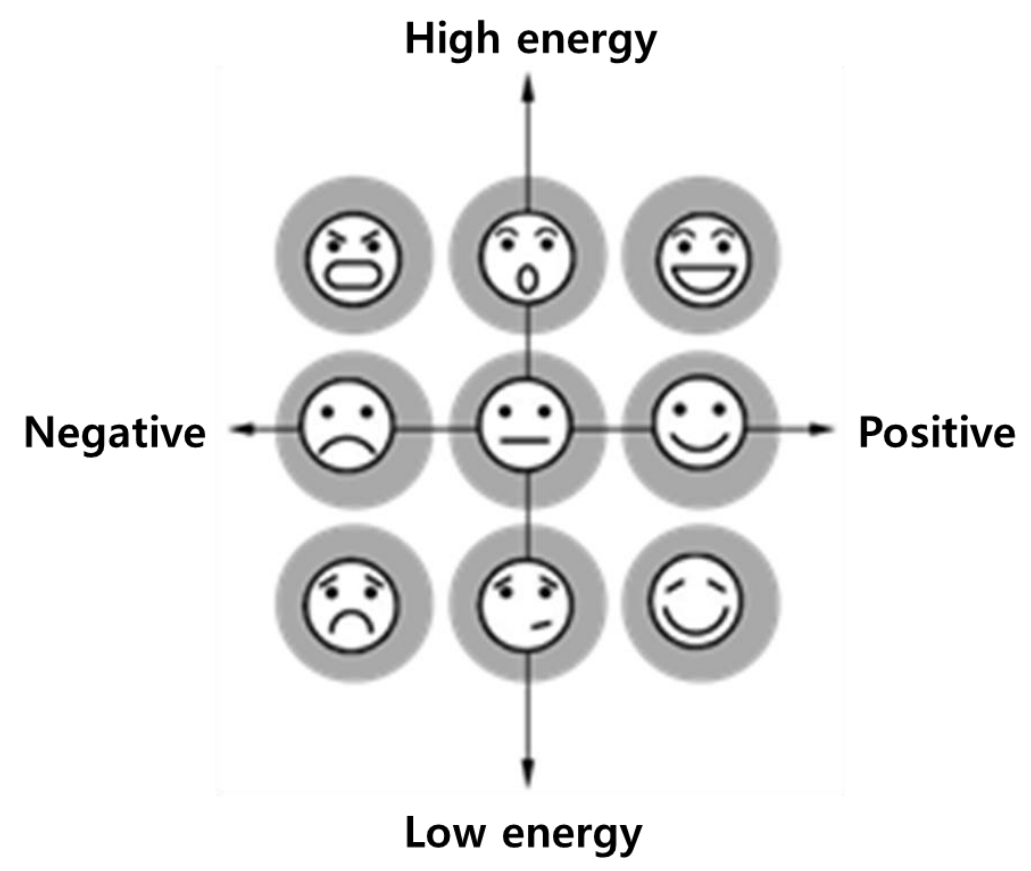

\section{Figure 1. Emotion Matrix Configured by Energy and Mood. Each Composition Indicates a Specific Feeling Figure}

The emotion library is composed of 9 regions where the amount of energy is divided into high, medium, and low energy and the degree of the mood is divided into positive, none, and negative emotion. For adding emotions selected by the user, according to the feeling selected by the user, the strength of the movement is determined. In order to express the 9 emotions in the selected motion, the emotion library is configured to carry the value of the parameter corresponding with energy levels with a positive / negative level of each region.

\subsection{Messenger Using Kinetic Typography}

In this paper, the emotional user interface provides the main movement which is often used in the mobile instant messenger. Select emotion after selecting motion' method provides a basic motion library that includes 6 motions to express user's feelings. 'Select motion after selecting emotion' method provides a motion library that is relevant to the selected emotion by the user. Basic motion library is separated into 6 movements: static rotation, static opacity, static shaking, static scale, line and circle, with each parameter as show in Table 1. And these are made in kinetic primitive motion API. 
Table 1. Primitive Motion Library and Its Parameters

\begin{tabular}{|c|c|}
\hline Motion & Parameters \\
\hline Static Rotation & Object, Angle, Duration time \\
\hline Static Opacity & Object, Opacity value, Duration time \\
\hline Static Shaking & Object, Size, duration time \\
\hline Static Scale & Object, Size, Duration time \\
\hline Line & Object, Direction, Length, Duration time \\
\hline Circle & Object, Angle, Radius, Duration time \\
\hline
\end{tabular}

In the future, a producer who makes motion libraries can provide a wide variety of motion libraries through a combination of primitive motion API using combined motion API. Users who use emotional user interface can also use a variety of motion libraries.

\subsection{Messenger using Kinetic Typography}

The emotional user interface provides two input methods: 'select emotion after selecting motion' method and 'Select motion after selecting emotion' method. 'Select emotion after selecting motion' method is where a kinetic typography effect is applied when the user selects a motion and then selects an emotion. 'Select motion after selecting emotion' method, on the other hand, is where a kinetic typography effect is applied when the user selects an emotion and then selects a motion.

3.3.1. Input Method: Select Emotion after Selecting Motion: 'Select emotion after selecting motion' input method follows this process: select user's feeling and apply values of emotion after select motion. Figure 2's (a), is an interface for selecting motions. The user can apply motion to text by selecting it in 6 motions of basic motion library. It can confirm kinetic typography applied selected motion and emotion at chat window in advance. (b) is an interface for selecting emotions. The user can select an emotion with emotion coordinates. Motion's values are changed according to the emotion selected by a user at (a). This controls the movement's degree of motion.

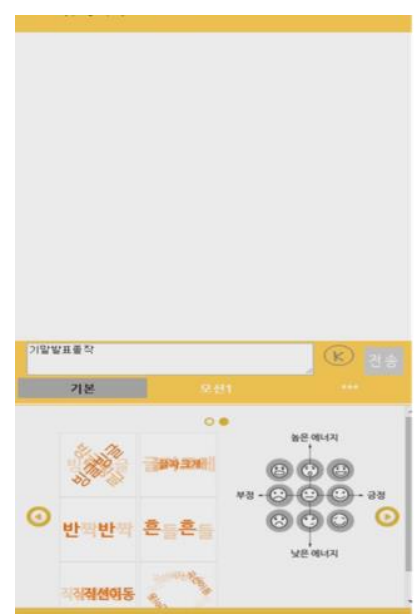

(a)

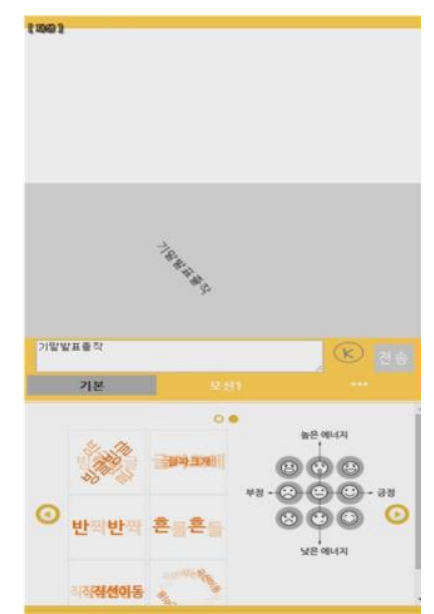

(b)

Figure 2. Select Emotion after Selecting Motion Input Method Interface 
3.3.2. Input Method: Select Motion after Selecting Emotion: Select emotion after selecting motion' input method follows this process: select user's feeling and apply values of emotion after select motion. Figure 3's (a), is an interface for selecting emotions. The user can select an emotion at emotion matrix. (b) is an interface for selecting motions. If the user selects any emotion, then the user can find a motion library that corresponds to the selected emotion at the motion area. The user can then apply motion to text by selecting it. It can confirm kinetic typography applied selected emotion and motion at the chat window in advance. The types of motions are changed according to the emotion selected by user at (a). This controls the kind of motions used.

3.3.3. Control Motion Parameters: It is an interface wherein a user can modify the parameters of the selected motion as shown in Figure 4. Because motions that are made through a motion library and emotion library that provided pre-production using 'Select emotion after selecting motion' method or 'Select motion after selecting emotion' method interface, it does not account for differences in the various feelings of the users. So, adjustment of parameters to modify a kinetic typography helps users to send an appropriate expression.

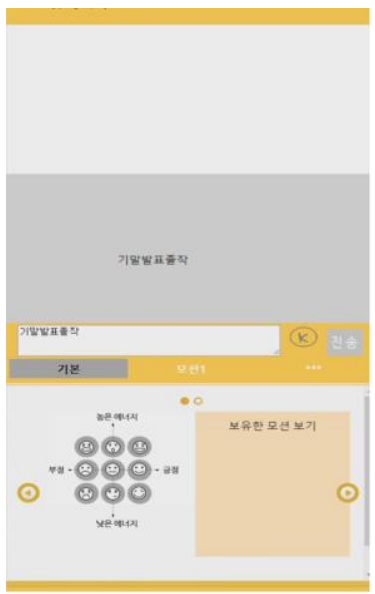

(a)

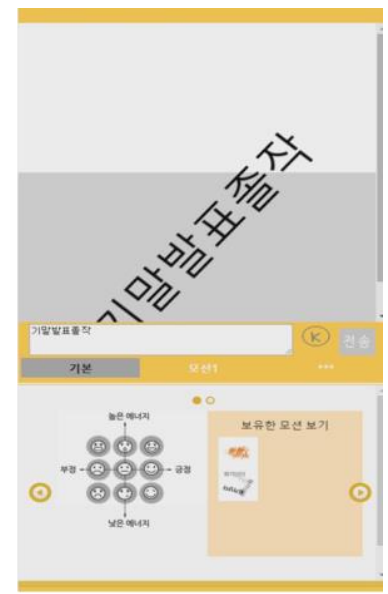

(b)

Figure 3. Select Motion After Selecting Emotion Input Method Interface

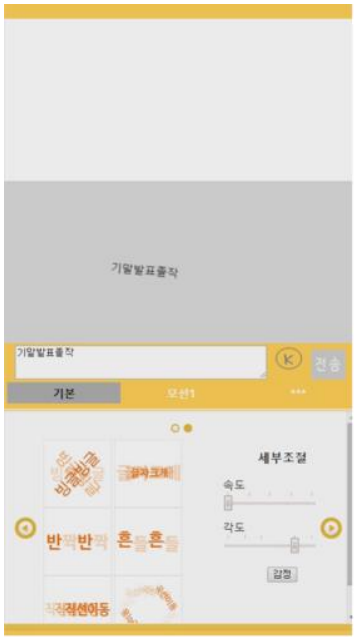

(a)

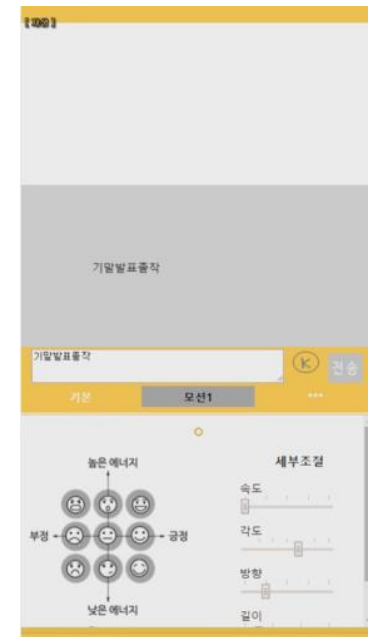

(b)

Figure 4. Modification Interface that Can Modify Parameters of the Selected Motion 


\section{Usability Evaluation}

In order to investigate the user interface preference for the two input methods proposed in this paper, we proceeded to the usability evaluation. Researchers measured the spending time required to transfer from selecting motion and selecting emotion using this user interface. Researchers also measured interface preferences, evaluating a total of 24 people- 8 of whom are teenagers, 8 in their twenties, and 8 in their thirties or over. 'Select motion after selecting emotion' method interface is called A. 'Select emotion after selecting motion' method interface, on the other hand, is called B.

First, the results of spending time on average are as follows: Interface A requires $46.5 \mathrm{sec}$ to teenagers, $34 \mathrm{sec}$ to twenties, and $55.5 \mathrm{sec}$ to thirties or over. Interface B requires $58.5 \mathrm{sec}$ to teenagers, $35 \mathrm{sec}$ to twenties, and $43.5 \mathrm{sec}$ to thirties or over Figure 5 . In total, interface A requires 43 seconds and B requires 45 seconds. Through this result, it is concluded that interface A and B require almost similar time. That is, they don't have any difference.

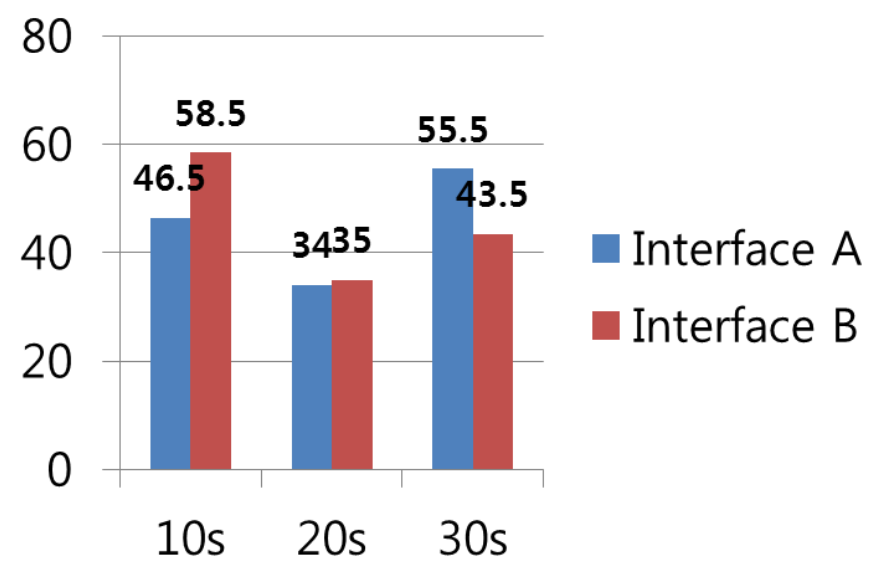

Figure 5. Result of Spending Time using Interface A and B

Finally, the results of preference are as follows: 14 people prefer interface A and 10 people prefer interface B Figure 6 . The preference of interface A is higher than interface $\mathrm{B}$, however, there is no huge difference. In conclusion, it is proven that both interfaces are necessary.

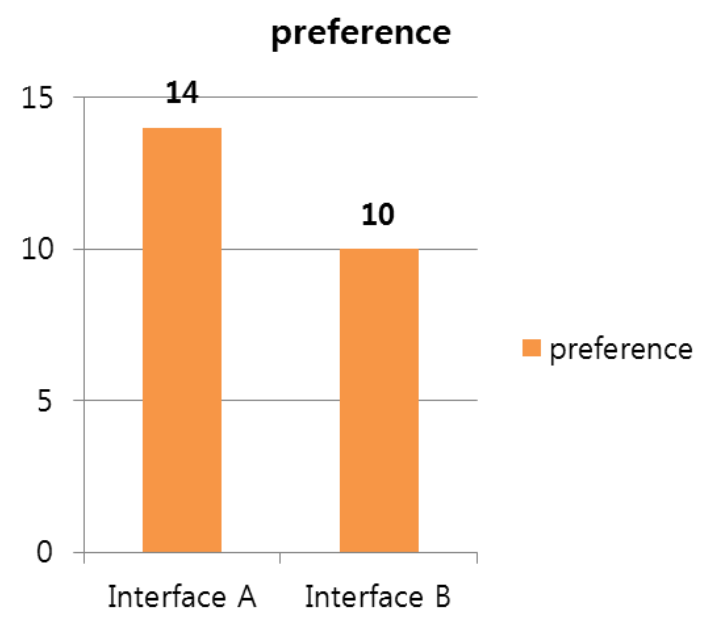

Figure 6. Results of Preference in Interface A and B 


\section{Conclusion}

The traditional text-based mobile instant messenger system is limited in efficiently transmitting feelings compared to direct dialogue. In this regard, kinetic typography can be effectively utilized as a method for passing emotions. In order to manufacture kinetic typography, a specialized animation program based on the timeline needs to be used. However, it can be difficult and take a long time to produce. Therefore, it is not a suitable production means of kinetic typography in the mobile instant messaging environment. In this paper, researchers have proposed an emotional user interface using kinetic typography that is made from kinetic motion API for mobile instant messenger, which helps the user to convey appropriate emotion with moving text.

In order to produce the emotional user interface, we used a kinetic motion API, which allows people to create kinetic typography without using specialized animation programs. Users can produce a text adding motion with the desired emotion easily and quickly by selecting motion in motion library and emotion in emotion matrix. Moreover, users can re-edit suitable motion to personal feelings by controlling the motion's parameters of the basic motion library. Thus, researchers expect that the concept of emotional user interface would be used to guide future studies providing personalized service.

\section{References}

[1] S. Ford, J. Forlizzi and S. Ishizaki, "Kinetic Typography: Issues in time-based presentation of text", ACM CHI 97, (1997).

[2] J. Lee, S. Jang, D. Kim, J. Wee, Y. Jang, I. Won, S. Ha and S. Jun, "Kinetic Typography Instant Messenger to Convey Emotion in Real Time Text-based Communication", KIISE, 2013, (2013)

[3] Y. A. Cho, J. H. Park and S. B. Lim, "Design a Primitive Motion API for developing the Kinetic Typography Motion”, HCI 2014, (2014).

[4] J. Lee, D. Kim, J. Wee, S. Jang, S. Ha and S. Jun, "Evaluating Pre-defined Kinetic Typography Effects to Convey Emotions", Journal of Korea Multimedia Society, vol. 17, no. 1, (2014).

[5] J. E. Kwak and S. B. Lim, "Design of Motion Library for using the Kinetic Typography in Messengers", KIISE vol. 39, no. 22, (2012).

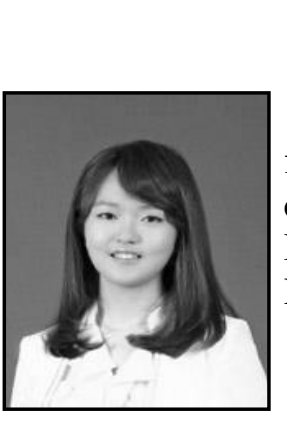

\section{Authors}

Seung-Ah Jung, She received her BS degree in Computer Science from Sookmyung Women's University, Korea, in 2014. She is currently attending the master's degree program in the Department of Multimedia Science at Sookmyung Women"s University in Korea. Her main research interests are web application and service, HTML5.

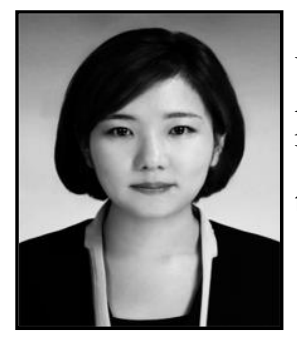

YoonAh Cho, She received her BS degree from SookMyung Women's University, Korea, in 2013. Since 2013, she has been pursuing an MS degree in Multimedia Science from SookMyung Women's University. Her main research interests are kinetic typography and user interface and smart service. 


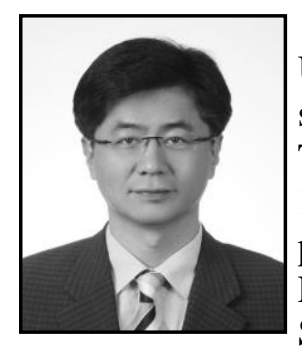

Soon-Bum Lim, He received his BS degree from Seoul National University, Korea, in 1982 and his MS and Ph.D degrees in computer science from KAIST (Korea Advanced Institute of Science and Technology), Korea, in 1983 and 1992, respectively. From 1989 to 1997, he was the engineering director for the font technology and printer division at Human Computer, Inc, and Trigem Computer, Inc. From 1997, he was an assistant professor in the Dept. of Computer Science at Konkuk University in Korea. Since 2001, he has been a professor in the Dept. of Multimedia Science at Sookmyung Women's University in Korea. His main research interests are computer graphics, web and mobile multimedia contents, user interface, and electronic publishing such as font, eBook, and XML documents. 